\title{
On cyclic steady states and elastic shakedown in diffusion-induced plasticity
}

\author{
Michaël Peigney
}

\begin{abstract}
This chapter is devoted to media in which plasticity and diffusion are coupled, such as electrode materials in lithium ion batteries. We present some recent results on the large time behavior of such media when they are submitted to cyclic chemo-mechanical loadings. Under suitable technical assumptions, we notably show that there is convergence towards a cyclic steady state in which the stress, the plastic strain rate, the chemical potential and the concentration of guest atoms are all periodic in time (with the same period as the applied loading). A special case of interest is that of elastic shakedown, which corresponds to the situation where the medium behaves elastically in the large time limit. We present general theorem that allow one to construct both lower and upper bounds of the set of loadings for which elastic shakedown occurs, in the spirit of Melan and Koiter theorems in classical plasticity. An illustrative example - for which all the relevant calculations can be done in closed-form - is presented.
\end{abstract}

\section{Introduction}

This chapter is devoted to cyclically loaded media in which plasticity and diffusion are coupled. An example of such media is electrode materials in lithium-ion batteries: in those batteries, the flow of electrons is the result of lithium ion diffusing in electrode particles (and in an electrolyte). The absorption of lithium in electrode particles produces some swelling, which in certain situations can be large enough to trigger plastic flow. In the media considered - of which electrode materials are an example - plastic flow and diffusion act as two concurrent dissipative processes. This results in a complex evolution problem in which the interplay between plastic flow and diffusion may lead to some unusual behavior. For instance, under a

Michaël Peigney

Lab Navier, Univ Gustave Eiffel, ENPC, CNRS, F-77447 Marne la Vallée, France, e-mail: michael.peigneyepolytechnique.org 
monotone loading, a particle may flow plastically for some time and later unloads elastically [1]. The main objective in this chapter is to study the large-time behavior of solutions to the evolution problem in the case of cyclic chemo-mechanical loadings. In classical plasticity (without diffusion), some general results are available [4, 5, 6] and fostered the development of direct methods aiming at determining the large-time response without resorting to step-by-step incremental analysis [20, 10, 21, 23]. A central question, which we address in this chapter, is to investigate whether similar general results can be established for media coupling plasticity with diffusion. The outline of this chapter is as follows: In Sect. 2 are derived the governing equations of the evolution problem. As an illustration, some finite element simulations on a model problem related to lithium-ion batteries are presented. In Sect. 3 are presented the main results on cyclic steady states in diffusion-induced plasticity. Under suitable technical assumptions, we notably show that any solution to the evolution problem converges towards a cyclic steady state in which the stress, the plastic strain rate, the chemical potential and the concentration of guest atoms are all periodic in time (with the same period as the applied loading). Sect. 4 focuses on elastic shakedown, defined as the special case where the plastic dissipation is bounded on the time interval $[0,+\infty$ ) (or, in more intuitive terms, that the medium behaves elastically in the large time limit). In classical plasticity, Melan and Koiter theorems deliver bounds on the set of loadings for which elastic shakedown occurs. Those theorems (Melan theorem especially) have been extended to several types of nonlinear behaviors, see e.g. [14, 15, 16, 17, 22, 7] for recent examples. Building on the results of Sect. 3 we show that both a Melan-type theorem and a Koiter-type theorem can be obtained for media coupling plasticity with diffusion. An illustrative example - for which all the relevant calculations can be done in closed-form is presented.

\section{Diffusion-induced plasticity in a cyclically loaded continuum}

\subsection{Conservation equations}

Consider a deformable continuum occupying a domain $\Omega$, in which guest atoms diffuse. The continuum is submitted to a body force $\mathbf{f}$. Tractions $\mathbf{T}$ are prescribed on a part $\Gamma_{T}$ of the boundary and displacements $\mathbf{U}$ are prescribed on $\Gamma_{u}=\partial \Omega-$ $\Gamma_{T}$. A normal flux $J$ of guest atoms is prescribed on a part $\Gamma_{J}$ of the boundary. On $\Gamma_{\mu}=\partial \Omega-\Gamma_{J}$, the chemical potential $\mu$ is prescribed to take a given value $M$. The functions $\mathbf{f}, \mathbf{U}, \mathbf{T}, M, J$ define the chemo-mechanical loading history. Those functions are assumed to be periodic in time, with the same period $T$. Functions that are periodic in time with a period $T$ as referred to as $T$-periodic in the following.

For the problem at hand, the two main conservation equations are the diffusion equation (expressing the mass conservation of guest atoms)

$$
\dot{c}+\operatorname{div} \mathbf{j}=0 \text { in } \Omega,
$$


and the equilibrium equation (assuming quasi-static evolutions)

$$
\operatorname{div} \sigma+\mathbf{f}=0 \text { in } \Omega .
$$

In (1) and (2), $c$ is the concentration of guest atoms, $\mathbf{j}$ is the flux and $\sigma$ is the stress. Eqs (1) and (2) are complemented by the boundary conditions

$$
\mu=M \text { on } \Gamma_{\mu}, \quad \mathbf{j} \cdot \mathbf{n}=J \text { on } \Gamma_{J}, \quad \sigma \cdot \mathbf{n}=\mathbf{T} \text { on } \Gamma_{T}, \quad \mathbf{u}=\mathbf{U} \text { on } \Gamma_{u} .
$$

\subsection{Constitutive equations}

The constitutive material is assumed to be elastic-plastic. Following the thermodynamic framework of [9] in small perturbations, the local state of the material is described by the total linearized strain $\varepsilon$, the (deviatoric) plastic strain $\varepsilon^{p}$ and the concentration $c$ of guest atoms. The free energy $w$ of the material is taken as a quadratic function of the form

$$
w\left(\varepsilon, \varepsilon^{p}, c\right)=\frac{1}{2}\left(\varepsilon-\varepsilon^{p}\right): \mathbb{L}:\left(\varepsilon-\varepsilon^{p}\right)+\frac{1}{2} k c^{2}+c \mathbf{A}:\left(\varepsilon-\varepsilon^{p}\right)+c \mu_{0}
$$

where the fourth-order symmetric tensor $\mathbb{L}$, the symmetric second-order tensors $\mathbf{A}$, the scalars $k$ and $\mu_{0}$ are material parameters satisfying

$$
\mathbb{L} \succ 0, \quad k>0, \quad k-\mathbf{A}: \mathbb{L}^{-1}: \mathbf{A}>0
$$

where the notation $\succ$ indicates that a tensor is positive definite. The requirements (5) ensure that $w$ is convex and guarantee the uniqueness of the evolution starting from a given initial state, as will be later discussed. From (4) we obtain the constitutive relations

$$
\sigma=\frac{\partial w}{\partial \varepsilon}=\mathbb{L}:\left(\varepsilon-\varepsilon^{p}\right)+c \mathbf{A}, \mu=\frac{\partial w}{\partial c}=\mu_{0}+k c+\mathbf{A}:\left(\varepsilon-\varepsilon^{p}\right) .
$$

It can be observed that the tensor $\mathbf{A}$ in (6) induces a two-way coupling between mechanics and diffusion. In particular, the concentration $c$ has a linear influence on the stress, in a way similar to thermal stress. Similarly, the chemical potential $\mu$ depends linearly on the elastic strain.

The constitutive equations (6) are complemented by a law of diffusion and a plasticity flow rule complying with the second law of thermodynamics. In more detail, the flux $\mathbf{j}$ of guest atoms is assumed to obey the relation

$$
\mathbf{j}=-\psi^{\prime}(\nabla \mu) \text {. }
$$

where $\psi$ is a positive, differentiable and strictly convex function such that $\psi(0)=0$. The special case of Fick's law (linear diffusion) corresponds to the situation $\psi=$ $\frac{1}{2} \mathbf{j} \cdot \mathbf{D} \cdot \mathbf{j}$ for some symmetric second order tensor $\mathbf{D} \succ 0$. 


\subsection{Illustrative example}

In order to illustrate the type of behavior that arises in the media considered, we present some finite-element simulations performed on a plane strain problem related to lithium-ion batteries. We consider a free-standing cylinder electrode particle with radius $R$. Cyclic lithiation-delithiation is achieved by applying a $T$-periodic flux

$$
J(t)=-\frac{R \omega}{4} H \sin \omega t
$$

on the boundary. In $(8), \omega$ and $H>0$ are given. The loading parameter $H$ in (8) is directly related to the maximum number of inserted lithium ions which is indeed equal to $-2 \pi R L \int_{0}^{T / 2} J(t) d t=\pi R^{2} L H$ where $L$ is the length of the cylinder in the $\mathbf{e}_{z}$ direction.

The free energy $w$ is taken as an isotropic version of (4), i.e.

$$
w\left(\varepsilon, \varepsilon^{p}, c\right)=\frac{1}{2} K(\operatorname{tr} \varepsilon)^{2}+G\left(\varepsilon^{d}-\varepsilon^{p}\right):\left(\varepsilon^{d}-\varepsilon^{p}\right)+\frac{1}{2} k c^{2}+a c \operatorname{tr} \varepsilon+c \mu_{0}
$$

where $\varepsilon^{d}$ is the deviatoric strain and $c$ is the concentration of lithium. In $(9), K, G$, $a$ and $\mu_{0}$ are material parameters. The constitutive relations (6) specialize as

$$
\sigma_{m}=K \operatorname{tr} \varepsilon+a c, \quad \mathbf{s}=2 G\left(\varepsilon^{d}-\varepsilon^{p}\right), \quad \mu=\mu_{0}+k c+a \operatorname{tr} \varepsilon,
$$

where $\sigma_{m}=(\operatorname{tr} \sigma) / 3$ is the hydrostatic stress and $\mathbf{s}$ is the deviatoric stress. The scalar material parameter $a$ in 9 accounts for the chemo-mechanical coupling. In particular, the ratio $-a / K$ can be interpreted as the volumetric expansion coefficient associated with the insertion of lithium.

Fick's law is adopted for diffusion, i.e.

$$
\mathbf{j}=\frac{D}{k} \nabla \mu
$$

where $D$ is the diffusion coefficient. A Von Mises plasticity model is adopted. The corresponding elasticity domain if defined by

$$
\frac{1}{2} \mathbf{s}: \mathbf{s} \leq \sigma_{Y}^{2}
$$

where $\sigma_{Y}$ is the yield strength.

The numerical results presented in the following have been obtained using the parameters $E=80 \mathrm{GPa}, v=0.3, \sigma_{Y}=50 \mathrm{MPa}, a=-2.72 \times 10^{-18} \mathrm{~J}, k=0.5 \times$ $10^{-13} \mathrm{~J} . \mathrm{m}^{3}, D=10^{-16} \mathrm{~m}^{2} / \mathrm{s}$. The bulk modulus $K$ and the shear modulus $G$ are related to Young's modulus $E$ and Poisson's ratio $v$ by the expressions $K=E / 3(1-$ $2 v)$ and $G=E / 2(1+v)$. In Figs 1 and 2 is shown the time evolution of the plastic strain field $\varepsilon^{p}$ for two different sets of loading parameters. For both simulations the initial value $\varepsilon^{p}(0)$ is non zero and taken as 


$$
\varepsilon^{p}(0)=0.003 \sin (\pi r / 2 R)\left(\mathbf{e}_{1} \otimes \mathbf{e}_{2}+\mathbf{e}_{2} \otimes \mathbf{e}_{1}\right)
$$

where $\left(\mathbf{e}_{1}, \mathbf{e}_{2}\right)$ is a reference orthonormal frame. Fig 1 corresponds to the loading parameters $H=100 \mathrm{~mol} / \mathrm{m}^{3}, \omega=10 / T_{0}$ where $T_{0}$ is a time scale defined by

$$
T_{0}=\frac{R^{2}}{D\left(1-\frac{a \tilde{a}}{k}\right)} \text { with } \tilde{a}=\frac{3 a}{3 K+4 G} .
$$

The map $\left\|\varepsilon^{p}(t)-\varepsilon^{p}(0)\right\|$ is shown at several time instants during the first two loading cycles. The plots in Fig. 1 suggest that the plastic strain stabilizes to a timeindependent field, i.e. that elastic shakedown occurs. Fig. 2 corresponds to the loading parameters $H=100 \mathrm{~mol} / \mathrm{m}^{3}$ and $\omega=20 / T_{0}$. The plots in Fig. 2 suggest that the plastic strain reaches a cyclic steady state, i.e. that cyclic plasticity occurs. Note that the same value of the parameter $H$ has been used in Figs. 1 and 2, which means that the same number of lithium is injected in the particle. The only difference is the charging rate. The loading rate is thus found to have an influence on the plastic response of the system, even though rate-independent plasticity is considered in the constitutive equations. This is a result of the chemo-mechanical coupling: the combined facts that diffusion is a rate-dependent process and that the stress depends on the local concentration entail that the mechanical response of the system is ratedependent.
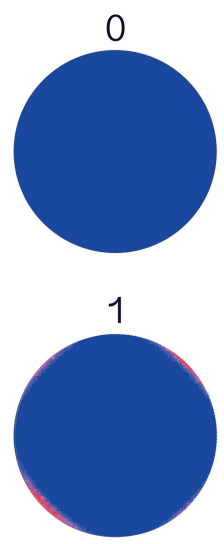

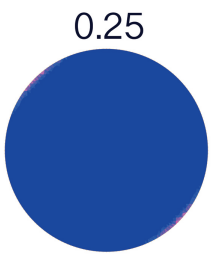

1.25

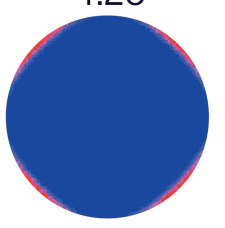

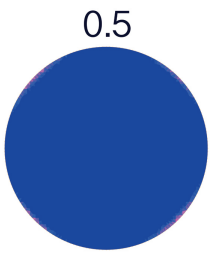

1.5

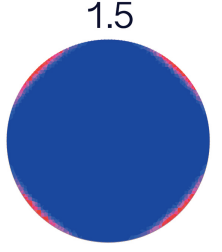

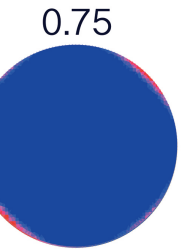

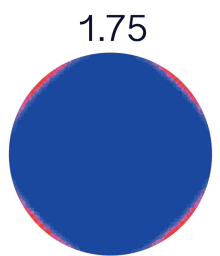

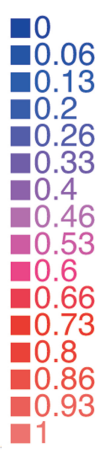

Fig. 1 Map of $\left\|\varepsilon^{p}(t)-\varepsilon^{p}(0)\right\|$ at several times instants $t / T$ for a cylinder particle under cyclic lithiation. Case $H=100 \mathrm{~mol} / \mathrm{m}^{3}, \omega=10 / T_{0}$. Reported values of $\left\|\varepsilon^{p}(t)-\varepsilon^{p}(0)\right\|$ are normalized.

In the electrochemical community, a quantity of interest is the charge-voltage response, relating the total charge $C$ of lithium ions in the electrode particle to the voltage $V$ on the surface of the particle. The charge $C$ is obtained from the con- 
centration field $c$ by $C=\int_{\Omega} c d \Omega$. The voltage $V$ is directly related to the chemical potential $\mu$ by $V=-\left(\mu-\mu_{0}\right) / e$ where $e$ is the elementary charge. Let $C(t)$ and $V(t)$ be the charge and voltage at time $t$, as obtained in the finite element simulations. The trajectories of $(C(t), V(t))$ in the $C-V$ plane are shown in Fig. 3 for the two loadings considered previously. It can be observed that $(C(t), V(t))$ converges towards a cyclic steady state. Some hysteresis is displayed in the cyclic steady state, even in the case $\omega=10 / T_{0}$ for which elastic shakedown occurs. Indeed, even if plastic flow vanishes in the elastic shakedown regime, there remains the diffusion-related dissipation corresponding to the cyclic insertion of lithium ions. By contrast, in the case $\omega=20 / T_{0}$, the diffusion-related dissipation and the plastic dissipation (due to cyclic plasticity) add up on the cyclic steady state. For designing lithium-ion batteries with improved electrochemical efficiency, it is beneficial to reduce the hysteresis in the $C-V$ response. In that regard, the shakedown regime is likely to be beneficial [1]. It can further be added that elastic shakedown is also beneficial for the mechanical fatigue behavior since elastic shakedown is typically associated with high-cycle fatigue - as opposed to cyclic plasticity which is associated with low-cycle fatigue [2].

The results in Figs. 1, 2 and 3 have been obtained for specific geometry, loading and material parameters. One can wonder to what extent those results are general. Considering a continuum of arbitrary shape submitted to a cyclic chemo-mechanical loading, the main questions to be addressed are

1. Is there always convergence to a steady state cycle ?

2. How does the steady state cycle (if exists) depend on the initial state ?

3. Is is possible to obtain a priori restrictions on the loading for ensuring that elastic shakedown occurs?

Regarding question 3 above, Melan theorem (also known as the static shakedown theorem) and Koiter theorem (also known as the kinematic shakedown theorem) come to mind [11, 24, 8]. In standard plasticity (without coupling with diffusion), those theorems provide bounds on the set of loadings for which elastic shakedown occurs. The classical reasoning used in the proof of those theorems consists in bounding the total dissipation on the time interval $[0, \infty)$. For the media considered here, there are two sources of dissipation, namely plasticity and diffusion. Bounding the total dissipation thus essentially means that there is no plastic flow and no diffusion in the large time limit. This is not really the situation of interest for applications such as lithium-ion batteries: one is instead interested in situations where there is no plastic flow in the large limit but diffusion still occurs as a result of cyclic lithiation, as in Fig. 1. In such situations, the total plastic dissipation on the time interval $[0, \infty)$ is bounded but the diffusion-related dissipation is not. There does not seem to be any obvious way to modify Melan's and Koiter's reasoning so as to bound only a part of the total dissipation (namely the plastic dissipation). As will shown in the 
following, shakedown theorems as desired can still be obtained at the price of first addressing the more general questions 1 and 2 listed above.
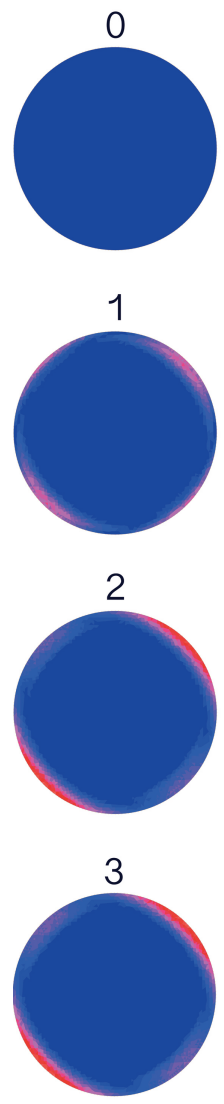

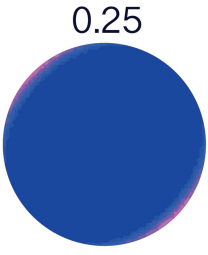

1.25

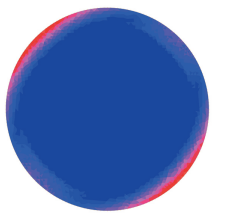

2.25

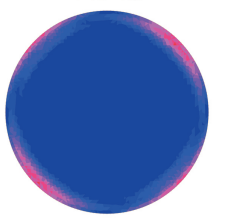

3.25

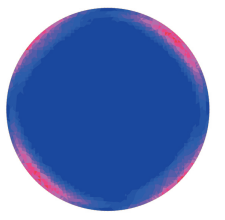

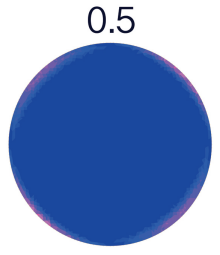

1.5

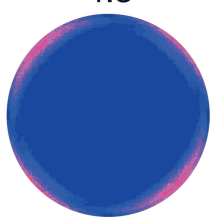

2.5

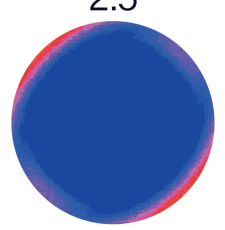

3.5

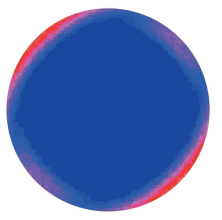

0.75

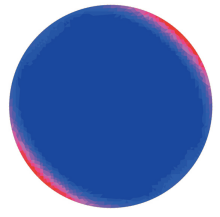

1.75

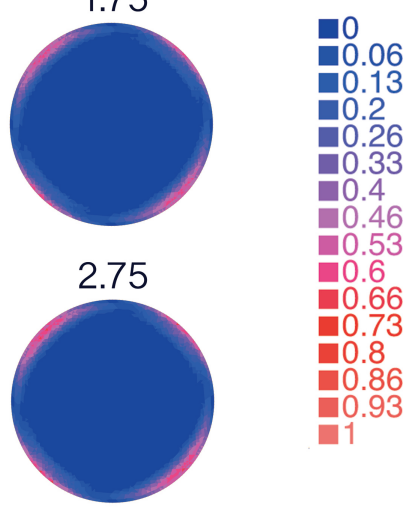

3.75

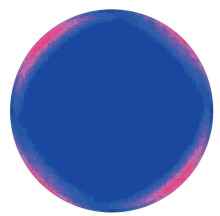

Fig. 2 Map of $\left\|\varepsilon^{p}(t)-\varepsilon^{p}(0)\right\|$ at several times instants $t / T$ for a cylinder particle under cyclic lithiation. Case $H=100 \mathrm{~mol} / \mathrm{m}^{3}, \omega=20 / T_{0}$. Reported values of $\left\|\varepsilon^{p}(t)-\varepsilon^{p}(0)\right\|$ are normalized.

\section{General results on steady state cycles}

In this section are presented some general results addressing questions 1-2 related to cyclic steady states in diffusion-induced plasticity. We first derive an evolution equation for the fields $(\sigma, \mu)$ and proceed to discuss some properties of that equation. As a preliminary, observe that the free energy $w\left(\varepsilon, \varepsilon^{p}, c\right)$ in (4) can be put in the form 
Fig. 3 Charge-Voltage response for $\omega=10 / T_{0}$ (blue curve) and $\omega=20 / T_{0}$ (red curve). The parameter $H$ is set to $H=100 \mathrm{~mol} / \mathrm{m}^{3}$.

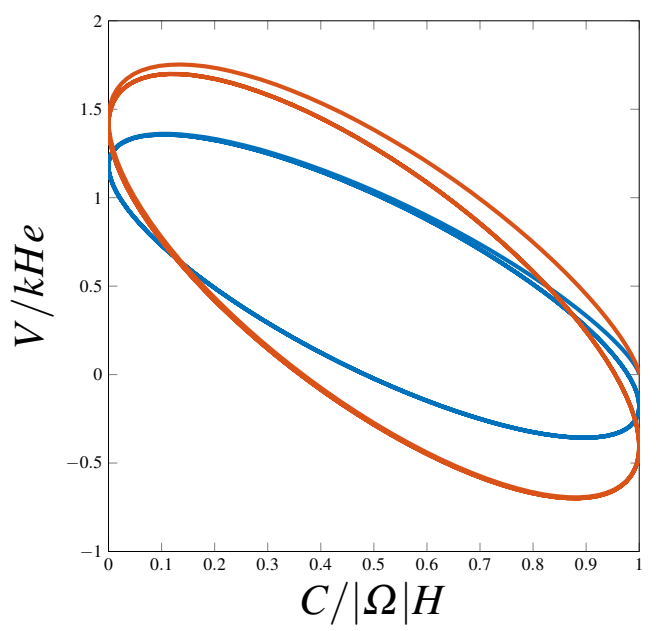

$$
w\left(\varepsilon, \varepsilon^{p}, c\right)=\frac{1}{2}\left(\varepsilon-\varepsilon^{p}, c\right): \mathbb{M}:\left(\varepsilon-\varepsilon^{p}, c\right)+c \mu_{0}
$$

where $\mathbb{M}$ is the symmetric operator defined by the relations

$$
\mathbb{M}:\left(\varepsilon-\varepsilon^{p}, c\right)=\left(\mathbb{L}:\left(\varepsilon-\varepsilon^{p}\right)+c \mathbf{A}, k c+\mathbf{A}:\left(\varepsilon-\varepsilon^{p}\right)\right) .
$$

for any $\varepsilon-\varepsilon^{p}$ and $c$. Under the assumptions $(5), \mathbb{M}$ can be verified to be positive definite. The operator $\mathbb{M}$ is thus invertible and its inverse $\mathbb{M}^{-1}$ is symmetric positive definite. For later reference, note that the constitutive relations (6) can be rewritten as

$$
\left(\varepsilon-\varepsilon^{p}, c\right)=\mathbb{M}^{-1}:\left(\sigma, \mu-\mu_{0}\right) .
$$

Setting $\mathbf{B}=\mathbf{A}: \mathbb{L}^{-1}$ and $k^{\prime}=k-\mathbf{A}: \mathbb{L}^{-1}: \mathbf{A}$, it can be calculated that

$$
\left.(\sigma, \mu): \mathbb{M}^{-1}:\left(\sigma^{\prime}, \mu^{\prime}\right)\right\rangle=\sigma: \mathbb{L}^{-1}: \sigma^{\prime}+\frac{1}{k^{\prime}}\left((\mathbf{B}: \sigma)\left(\mathbf{B}: \sigma^{\prime}\right)+\mu \mu^{\prime}-\mu^{\prime} \mathbf{B}: \sigma-\mu \mathbf{B}: \sigma^{\prime}\right)
$$

for any $(\sigma, \mu)$ and $\left(\sigma^{\prime}, \mu^{\prime}\right)$. To simplify the presentation, we will consider the case of viscoplasticity: the flow rule takes the form $\dot{\varepsilon}^{p}=\phi^{\prime}(\sigma)$ where $\phi$ is a positive, strictly convex, differentiable function of the deviatoric stress and such that $\phi(0)=0$. The elasticity domain of the material is denoted by $\mathscr{C}$.

\subsection{Evolution equation for the stress and the chemical potential}

The fields $(\sigma, \mu)$ are assumed to live in a closed subspace of $H(\operatorname{div} ; \Omega) \times H^{1}(\Omega)$. Since $\mathbb{M}^{-1} \succ 0, \mathscr{H}$ is a Hilbert space for the scalar product $\langle\cdot, \cdot\rangle$ defined by 


$$
\left\langle(\sigma, \mu),\left(\sigma^{\prime}, \mu^{\prime}\right)\right\rangle=\int_{\Omega}(\sigma, \mu): \mathbb{M}^{-1}:\left(\sigma^{\prime}, \mu^{\prime}\right) d \Omega
$$

We denote $\mathscr{K}(\mathbf{f}, \mathbf{T}, M)$ the set of stress and chemical potential fields that are compatible with data $(\mathbf{f}, \mathbf{T}, M)$, i.e

$$
\mathscr{K}(\mathbf{f}, \mathbf{T}, M)=\left\{(\sigma, \mu) \in \mathscr{H}: \operatorname{div} \sigma+\mathbf{f}=0 \text { in } \Omega, \sigma \cdot \mathbf{n}=\mathbf{T} \text { on } \Gamma_{T}, \mu=M \text { on } \Gamma_{\mu}\right\} .
$$

Let $(\sigma, \mu)$ be the stress and chemical potential in a given evolution satisfying the governing equations described in Sect. 2. We have $(\sigma, \mu) \in \mathscr{K}(\mathbf{f}, \mathbf{T}, M)$ and it can be verified that

$$
\langle(\dot{\sigma}, \dot{\mu}),(\rho, v)\rangle=-\int_{\Omega}\left(\phi^{\prime}(\sigma): \rho+\psi^{\prime}(\nabla \mu) \cdot \nabla v\right) d \Omega-\int_{\Gamma_{J}} J v d S \quad \forall(\rho, v) \in \mathscr{K}_{0} ;
$$

with $\mathscr{K}_{0}=\left\{(\sigma, \mu) \in \mathscr{H}: \operatorname{div} \sigma=0\right.$ in $\Omega, \sigma \cdot \mathbf{n}=0$ on $\Gamma_{T}, \mu=0$ on $\left.\Gamma_{\mu}\right\}$. Eq. can be interpreted as an evolution equation for the stress and chemical potential fields $(\sigma, \mu)$.

\subsection{Contraction properties}

The norm in $\mathscr{H}$ is denoted by $\|\cdot\|$, i.e. $\|(\sigma, \mu)\|^{2}=\int_{\Omega}(\sigma, \mu): \mathbb{M}^{-1}:(\sigma, \mu) d \Omega$. The two following lemmas can be proved [18]:

Lemma 1. Let $\Sigma=(\sigma, \mu)$ and $\Sigma^{\prime}=\left(\sigma^{\prime}, \mu^{\prime}\right)$ be two solutions of (16). Then

$$
\left\|\Sigma\left(t_{2}\right)-\Sigma^{\prime}\left(t_{2}\right)\right\| \leq\left\|\Sigma\left(t_{1}\right)-\Sigma^{\prime}\left(t_{1}\right)\right\| \text { for all } t_{1}<t_{2} .
$$

Lemma 2. Let $\Sigma=(\sigma, \mu)$ and $\Sigma^{\prime}=\left(\sigma^{\prime}, \mu^{\prime}\right)$ be two solutions of (16) such that $\left\|\Sigma(t)-\Sigma^{\prime}(t)\right\|=\left\|\Sigma\left(t_{1}\right)-\Sigma^{\prime}\left(t_{1}\right)\right\|$ for all $t_{1} \leq t$. Then

$$
\phi^{\prime}(\sigma(\mathbf{x}, t))=\phi^{\prime}\left(\sigma^{\prime}(\mathbf{x}, t)\right) \text { for } t_{1} \leq t .
$$

Moreover, there exists a time-independent stress field $\rho$ and a constant $\eta$ such that $(\rho, \eta) \in \mathscr{K}_{0}$ and

$$
\sigma(\mathbf{x}, t)-\sigma^{\prime}(\mathbf{x}, t)=\rho(\mathbf{x}), \mu(\mathbf{x}, t)-\mu^{\prime}(\mathbf{x}, t)=\eta \text { for } t_{1} \leq t .
$$

Lemma 1 means that the distance between two solutions decreases with time. It notably implies the uniqueness of the solution for a given initial state. Lemma 2 means that if the distance between two solutions is constant then the difference between those solutions is constant (which is not obvious at first sight). 


\subsection{Main results}

We are now in a position to prove the main results regarding $T$-periodic solutions to the evolution equation (16). Although such $T$-periodic solutions are not unique, they are quite similar to one another. More precisely, we have the following

Theorem 1. Let $\Sigma=(\sigma, \mu)$ and $\Sigma^{\prime}=\left(\sigma^{\prime}, \mu^{\prime}\right)$ be two $T$-periodic solutions of (16). Then

$$
\phi^{\prime}(\sigma(\mathbf{x}, t))=\phi^{\prime}\left(\sigma^{\prime}(\mathbf{x}, t)\right) \text { for } t_{1} \leq t
$$

Moreover, there exists a time-independent stress field $\rho$ and a constant $\eta$ such that $(\rho, \eta) \in \mathscr{K}_{0}$ and

$$
\sigma(\mathbf{x}, t)-\sigma^{\prime}(\mathbf{x}, t)=\rho(\mathbf{x}), \mu(\mathbf{x}, t)-\mu^{\prime}(\mathbf{x}, t)=\eta
$$

Proof. The result follows from Lemmas 1 and 2. Let $\Sigma=(\sigma, \mu)$ and $\Sigma^{\prime}=\left(\sigma^{\prime}, \mu^{\prime}\right)$ be two $T$-periodic solutions of $(16)$. By Lemma 1 we have

$$
\left\|\Sigma(T)-\Sigma^{\prime}(T)\right\| \leq\left\|\Sigma(t)-\Sigma^{\prime}(t)\right\| \leq\|\Sigma(0)-\Sigma(0)\|
$$

for all $t \in[0, T]$. Since $\Sigma$ and $\Sigma^{\prime}$ are $T$-periodic, we have $\left\|\Sigma(T)-\Sigma^{\prime}(T)=\right\| \Sigma(0)-$ $\Sigma(0) \|$. Hence (17) becomes $\left\|\Sigma(t)-\Sigma^{\prime}(t)\right\|=\|\Sigma(0)-\Sigma(0)\|$ for all $t \in[0, T]$. Applying Lemma 2 proves the claim. (16):

We now arrive at the main result on the large time convergence of solutions to

Theorem 2. Assume that there exists a T-periodic solution to (16) and that the dimension of $\mathscr{H}$ is finite. For any solution $\Sigma(t)$ of $(16)$ on $[0, \infty)$, there exists a $T$-periodic solution $\Sigma^{\prime}$ of $(16)$ such that

$$
\Sigma(t) \rightarrow \Sigma^{\prime}(t) \text { as } t \rightarrow \infty
$$

Proof. We will make use of Opial's lemma [13], which is a general result in Hilbert spaces that reads as follows:

Let $F$ be a nonempty subset of $\mathscr{H}$ and $\left\{u_{n}\right\}$ a sequence in $\mathscr{H}$ such that :

(i) $\left\{\left\|u_{n}-f\right\|\right\}$ converges for all $f \in F$,

(ii) the limit of every (weakly) convergent subsequence of $\left\{u_{n}\right\}$ is in $F$.

Then the whole sequence $\left\{u_{n}\right\}$ (weakly) converges to a point of $F$. 
Here we only consider the simplified situation where the dimension of $\mathscr{H}$ is finite, so that weak and strong convergences coincide. Considering an arbitrary solution $\Sigma(t)$ of $[16)$, we wish to apply Opial's lemma to the sequence $\left\{u_{n}\right\}=\{\Sigma(n T)\}$ and to the set $F$ formed by the initial values of $T$-periodic solutions to (16), i.e. $F=\{\tilde{\Sigma}(0): t \mapsto \tilde{\Sigma}(t)$ is a $T$-periodic solution of $(16)\}$. To that purpose, the main effort consists in checking assumptions $(i)$ and (ii) in Opial's lemma.

Let us begin with assumption $(i)$. Consider $f \in F$ and let $\Sigma^{\prime}$ be a $T$-periodic solution of (16) such that $f=\Sigma^{\prime}(0)$. Lemma 1 gives

$$
\left\|\Sigma((n+1) T)-\Sigma^{\prime}((n+1) T)\right\| \leq\left\|\Sigma(n T)-\Sigma^{\prime}(n T)\right\| .
$$

Since $\Sigma^{\prime}$ is $T$ - periodic, we have $\Sigma(n T)=\Sigma(0)=f$ for all $n$. Hence $(18)$ becomes $\left\|u_{n+1}-f\right\| \leq\left\|u_{n}-f\right\|$. The positive sequence $\left\{\left\|u_{n}-f\right\|\right\}$ is decreasing. It follows that $\left\{\left\|u_{n}-f\right\|\right\}$ converges to a limit as $n \rightarrow \infty$, which shows that assumption $(i)$ is verified.

We now proceed with assumption $(i i)$. Let $\left\{u_{n_{k}}\right\}$ be a converging subsequence of $\left\{u_{n}\right\}$ and denote its limit by $g$. Denoting by $\Sigma^{*}(t)$ the solution of $(16)$ for the initial condition $\Sigma^{*}(0)=g$, we wish to show that $\Sigma^{*}(t)$ is $T$-periodic. We consider a fixed $t$ in the interval $[0, T]$ in what follows. Lemma 1 gives

$$
\left\|\Sigma\left(n_{k} T+t\right)-\Sigma^{*}(t)\right\| \leq\left\|\Sigma\left(n_{k} T\right)-\Sigma^{*}(0)\right\|=\left\|u_{n_{k}}-g\right\| \underset{k \rightarrow \infty}{\longrightarrow} 0 .
$$

Thus

$$
\Sigma\left(n_{k} T+t\right) \underset{k \rightarrow \infty}{\longrightarrow} \Sigma^{*}(t)
$$

Let $\Sigma^{\prime}$ be a $T$-periodic solution to (16). Lemma 1 yields

$$
\left\|\Sigma\left(n_{k+1} T\right)-\Sigma^{\prime}\left(n_{k+1} T\right)\right\| \leq\left\|\Sigma\left(n_{k} T+t\right)-\Sigma^{\prime}\left(n_{k} T+t\right)\right\| \leq\left\|\Sigma\left(n_{k} T\right)-\Sigma^{\prime}\left(n_{k} T\right)\right\|
$$

Setting $f=\Sigma^{\prime}(0) \in F$ and noting that $\Sigma^{\prime}\left(n_{k} T+t\right)=\Sigma^{\prime}(t)$, 20. becomes

$$
\left\|\Sigma\left(n_{k+1} T\right)-f\right\| \leq\left\|\Sigma\left(n_{k} T+t\right)-\Sigma^{\prime}(t)\right\| \leq\left\|\Sigma\left(n_{k} T\right)-f\right\| .
$$

By $(i)$, both the left and the right terms in 21) converge to a limit $A$ as $k \rightarrow \infty$. It follows that

$$
\left\|\Sigma\left(n_{k} T+t\right)-\Sigma^{\prime}(t)\right\| \underset{k \rightarrow \infty}{\longrightarrow} A .
$$

Combining (19) and 222 shows that $\left\|\Sigma^{*}(t)-\Sigma^{\prime}(t)\right\|=A$ for all $t$ in $[0, T]$. Lemma 2 implies that $\Sigma^{*}(t)-\Sigma^{\prime}(t)$ is time-independent. Hence $\Sigma^{*}(t)$ is $T$-periodic, i.e. $\Sigma^{*}(0)=g \in F$. Assumption (ii) is thus verified.

Applying Opial's lemma shows that there exists a $T$-periodic solution $\Sigma^{*}(t)$ such that $\Sigma(n T) \rightarrow \Sigma^{*}(0)$ as $n \rightarrow \infty$. By Lemma 1, it follows that $\Sigma(t)-\Sigma^{*}(t) \rightarrow 0$ as $t \rightarrow \infty$.

Theorems 1 and 2 allow one to provide some answers to questions 1-2 listed in Sect. 2 regarding cyclic steady states in diffusion-induced plasticity. Theorem 2 indeed implies that the stress $\sigma$, the plastic strain rate $\dot{\varepsilon}^{p}$ and the chemical potential 
$\mu$ converge towards a cyclic steady state as $t \rightarrow \infty$. Using the constitutive relations (7) and (14), it follows that the flux $\mathbf{j}$ and the concentration $c$ also converge towards a cyclic steady state. Since there is no uniqueness of $T$-periodic solutions to (16), the cyclic steady state depends on the initial state. However, Theorem 1 shows that some features of the cyclic steady state are unique, namely the stress rate, the plastic strain rate and the chemical potential (up to a constant). Using again (7) and (14), the flux and the concentration rate on the cyclic steady state are also unique, i.e. independent of the initial state.

Let us denote by $\dot{\varepsilon}_{\infty}^{p}$ the plastic strain rate on the cyclic steady steady state. The facts that $\dot{\varepsilon}_{\infty}^{p}$ is $T$-periodic and uniquely defined imply that there is either elastic shakedown, cyclic plasticity or ratchetting - with the exclusion of any other regime. Moreover, for a given structure, the type of asymptotic plastic behavior (i.e elastic shakedown, cyclic plasticity or ratchetting) is only determined by the loading, independently of the initial state. In a similar fashion, diffusion-related quantities reach a cyclic steady state which is largely independent of the initial state. In particular, for electrode particles in lithium-ion batteries, the charge-voltage response reaches a cyclic steady state which is uniquely defined (possibly up to a translation in the $C-V$ plane, depending on the type of chemical boundary conditions).

In such conditions, it makes sense to establish Bree-like diagrams mapping the space of load parameters to path-independent properties such as the type of asymptotic behavior, the plastic dissipation or the diffusion-related dissipation on the steady state cycle.

\section{Shakedown theorems in diffusion-induced plasticity}

Let us consider the fictitious purely elasto-diffusive problem obtained by discarding plastic flow in the original problem. As a special case of (16), the stress and chemical potential in the diffusive elasto-diffusive problem satisfy

$$
\langle(\dot{\sigma}, \dot{\mu}),(\rho, v)\rangle=-\int_{\Omega} \psi^{\prime}(\nabla \mu) \cdot \nabla v d \Omega-\int_{\Gamma_{J}} J v d S \quad \forall(\rho, v) \in \mathscr{K}_{0} .
$$

Periodic solutions to (23) play a central role in formulating shakedown conditions in diffusion-induced plasticity, as will be later demonstrated. Periodic solutions to (23) are not uniquely defined. As a particular case of Theorem 1, two periodic solutions of 23) differ by a time independent stress field $\rho$ and a constant $\eta$ such that $(\rho, \eta) \in$ $\mathscr{K}_{0}$. Conversely, if $(\rho, \eta) \in \mathscr{K}_{0}$ and $\left(\sigma^{E}, \mu^{E}\right)$ is a particular $T$-periodic solution to 23) then $\left(\sigma^{E}, \mu^{E}\right)+(\rho, \eta)$ can easily be verified to be a $T$-solution to 23). The whole set of $T$-periodic solutions to 23 is thus an affine space than can be written as

$$
\left\{\left(\sigma^{E}, \mu^{E}\right)+(\rho, \eta):(\rho, \eta) \in \mathscr{K}_{0}\right\}
$$

where $\left(\sigma^{E}, \mu^{E}\right)$ denotes any given $T$-periodic solution to 23 . 


\subsection{Static shakedown theorem}

Assume that elastic shakedown occurs in the original problem involving diffusioninduced plasticity and denote by $\left(\sigma_{\infty}, \mu_{\infty}\right)$ the stress and the chemical potential in the cyclic steady state. The fields $\left(\sigma_{\infty}, \mu_{\infty}\right)$ form a $T$-periodic solution to (16). Elastic shakedown corresponds to the situation where $\dot{\varepsilon}_{\infty}^{p}=\phi^{\prime}\left(\sigma_{\infty}\right)=0$, hence we have

$$
\left\langle\left(\dot{\sigma}_{\infty}, \dot{\mu}_{\infty}\right),(\rho, v)\right\rangle=-\int_{\Omega} \psi^{\prime}\left(\nabla \mu_{\infty}\right) \cdot \nabla v d \Omega-\int_{\Gamma_{J}} J v d S \quad \forall(\rho, v) \in \mathscr{K}_{0} ;
$$

Comparing 25] with 23 shows that $\left(\sigma_{\infty}, \mu_{\infty}\right)$ is a $T$-periodic solution to the elasto-diffusive problem (23). Conversely, assume there exists a $T$-periodic solution $(\tilde{\sigma}, \tilde{\mu})$ to the elasto-diffusive 23) such that $\phi^{\prime}(\tilde{\sigma})=0$. It can be directly checked that $(\tilde{\sigma}, \tilde{\mu})$ is also a $T$-periodic to the equation $[16$. Noting that the associated plastic strain is zero and using Theorem 1, we obtain that the plastic strain rate vanishes for any $T$-periodic solution to (16), i.e. in any cyclic steady state. Consequently, elastic shakedown is characterized by the existence of a $T$-periodic solution $(\tilde{\sigma}, \tilde{\mu})$ to (16) such that $\phi^{\prime}(\tilde{\sigma})=0$, i.e. such that $\tilde{\sigma} \in \mathscr{C}$. Using 24 , we can formulate the following result

Theorem 3. If there exists a time-independent stress field $\rho \in \mathscr{A}_{0}$ such that $\sigma^{E}(\mathbf{x}, t)+\rho(\mathbf{x}) \in \mathscr{C}$ for all $\mathbf{x} \in \Omega$ and $t \in[0, T]$, then elastic shakedown occurs (whatever the initial state is).

In Theorem $3, \mathscr{A}_{0}$ is the space of self-equilibrated stress fields, i.e.

$$
\mathscr{A}_{0}=\left\{\rho: \operatorname{div} \rho=0 \text { in } \Omega, \rho . \mathbf{n}=0 \text { on } \Gamma_{T}\right\} .
$$

Although the statement of Theorem 3 is similar to Melan theorem, we emphasize that its proof largely differs from the standard proof used in Melan theorem for pure plasticity. Theorem 3 motivates the introduction of a 'static security coefficient' $m_{S}$ as

$$
\begin{aligned}
& m_{S}=\sup \left\{m: \text { there exists } \rho \in \mathscr{A}_{0}\right. \text { such that } \\
& \left.\qquad \rho(\mathbf{x})+m \sigma^{E}(\mathbf{x}, t) \in \mathscr{C} \text { for all }(\mathbf{x}, t) \in \Omega \times[0, T]\right\} .
\end{aligned}
$$

The value of $m_{S}$ indeed determines the shakedown behavior according to the following rules

$$
\left\{\begin{array}{l}
m_{S}>1 \Longrightarrow \text { shakedown occurs } \\
m_{S}<1 \Longrightarrow \text { shakedown does not occur }
\end{array}\right.
$$

Lower bounds on $m_{S}$ can be obtained by using Theorem 3 with particular stress fields in $\mathscr{A}_{0}$. 


\subsection{Kinematic shakedown theorem}

Upper bounds on $m_{S}$ can be obtained by convex duality, as used for instance by [3, 12]. Let $\mathscr{A}$ be the set of triplets $\left(m, \rho^{*}, \tilde{\sigma}\right)$ such that $\rho^{*}(\mathbf{x})$ is a time-independent stress field in $\mathscr{A}_{0}$ and $\tilde{\sigma}(\mathbf{x}, t) \in \mathscr{C}$ for all $(\mathbf{x}, t)$. From 26 we have

$$
\begin{aligned}
m_{S}= & \sup _{\left(m, \rho^{*}, \tilde{\sigma}\right) \in \mathscr{A},} m \\
& \rho^{*}+m \sigma^{E}=\tilde{\sigma}
\end{aligned}
$$

This is a constrained maximization problem over the convex set $\mathscr{A}$. Denote by $\mathscr{L}$ the corresponding lagrangian, i.e.

$$
\mathscr{L}\left(m, \rho^{*}, \tilde{\sigma} ; \mathbf{d}\right)=m+\int_{\Omega} \int_{0}^{T} \mathbf{d}(\mathbf{x}, t):\left(\tilde{\sigma}(\mathbf{x}, t)-\rho^{*}(\mathbf{x})-m \sigma^{E}(\mathbf{x}, t)\right) d \Omega d t
$$

We have

$$
m_{S}=\sup _{\left(m, \rho^{*}, \tilde{\boldsymbol{\sigma}}\right) \in \mathscr{A} \mathbf{d}} \inf _{\mathbf{L}} \mathscr{L}\left(m, \rho^{*}, \tilde{\boldsymbol{\sigma}} ; \mathbf{d}\right)
$$

From the min-max inequality

$$
\sup _{\left(m, \boldsymbol{\rho}^{*}, \tilde{\boldsymbol{\sigma}}\right) \in \mathscr{A}} \inf _{\mathbf{d}} \mathscr{L} \leq \inf _{\mathbf{d}} \sup _{\left(m, \boldsymbol{\rho}^{*}, \tilde{\boldsymbol{\sigma}}\right) \in \mathscr{A}} \mathscr{L}
$$

we get

$$
m_{S} \leq m_{K}
$$

where

$$
m_{K}=\inf _{\mathbf{d}} \sup _{\left(m, \rho^{*}, \tilde{\sigma}\right) \in \mathscr{A}} \mathscr{L}\left(m, \rho^{*}, \tilde{\sigma} ; \mathbf{d}\right) .
$$

Rewriting the coefficient $m_{K}$ in a more explicit form [19] leads to

$$
m_{K}=\inf \left\{\int_{\Omega} \int_{0}^{T} \mathscr{D}(\mathbf{d}(\mathbf{x}, t)) d \Omega d t: \int_{0}^{T} \int_{\Omega} \mathbf{d}: \sigma^{E} d \Omega d t=1 \text { and } \int_{0}^{T} \mathbf{d} d t \in \mathscr{B}_{0}\right\}
$$

with $\mathscr{D}(\mathbf{d})=\sup _{\sigma \in \mathscr{C}} \sigma: \mathbf{d}$ and

$$
\mathscr{B}_{0}=\left\{\left(\nabla \mathbf{u}+\nabla^{t} \mathbf{u}\right) / 2: \mathbf{u}=0 \text { on } \Gamma_{u}\right\} .
$$

If $m_{K}<1$ then by (30) and 27) we can conclude that shakedown does not occur. A necessary condition for shakedown is thus that $m_{K} \geq 1$. We can thus formulate the following

Theorem 4. If shakedown occurs, then $1 \leq \int_{\Omega} \int_{0}^{T} \mathscr{D}(\mathbf{d}(\mathbf{x}, t)) d \Omega d t$ for any history $\mathbf{d}(\mathbf{x}, t)$ such that $\int_{0}^{T} \int_{\Omega} \mathbf{d}: \sigma^{E} d \Omega d t=1$ and $\int_{0}^{T} \mathbf{d} \in \mathscr{B}_{0}$. 
In practice, an upper bound $m_{K}^{+}$on $m_{K}$ (and therefore on $m_{S}$ ) can be obtained by constructing specific strain histories satisfying the requirements $\int_{0}^{T} \int_{\Omega} \mathbf{d}$ : $\sigma^{E} d \Omega d t=1$ and $\int_{0}^{T} \mathbf{d} \in \mathscr{B}_{0}$.

\subsection{Illustrative example}

As an illustration of the shakedown theorems, consider the example introduced in Sect. 2.3. A $T$ - periodic solution $\left(\sigma^{E}, \mu^{E}, \mathbf{u}^{E}, c^{E}\right)$ to the corresponding purely elasto-diffusive problem has been calculated in closed-form in [18]. Denoting by $\mathfrak{R}$ (resp. I) the real (resp. imaginary) part of a complex valued quantity, we have in particular

$$
\sigma^{E}(\mathbf{x}, t)=\Re\left(e^{i \omega t} \frac{G \tilde{a} H}{2}\left(\hat{\sigma}_{r} \mathbf{e}_{r} \otimes \mathbf{e}_{r}+\hat{\sigma}_{\theta} \mathbf{e}_{\theta} \otimes \mathbf{e}_{\theta}+\hat{\sigma}_{z} \mathbf{e}_{z} \otimes \mathbf{e}_{z}\right)\right)
$$

where

$$
\hat{\sigma}_{r}=1-\frac{\hat{R}}{\hat{r}} \frac{J_{1}(\hat{r})}{J_{1}(\hat{R})}, \quad \hat{\sigma}_{\theta}=1+\frac{\hat{R}}{\hat{r}} \frac{J_{1}(\hat{r})}{J_{1}(\hat{R})}-\frac{\hat{R} J_{0}(\hat{r})}{J_{1}(\hat{R})}, \quad \hat{\sigma}_{z}=2 v-\frac{\hat{R} J_{0}(\hat{r})}{J_{1}(\hat{R})}
$$

and

$$
\hat{r}=\hat{\lambda} r, \quad \hat{R}=\hat{\lambda} R, \quad \hat{\lambda}=e^{i \frac{3 \pi}{4}} \sqrt{\frac{\omega}{D(1-a \tilde{a} / k)}} .
$$

In (32), $J$ is the Bessel function of the first kind. The scalar $\tilde{a}$ in $(33)$ is defined as in (12).

Let us denote by $\mathbf{s}^{E}$ the deviatoric part of $\sigma^{E}$. Using Theorem 3 with $\rho=0$, we can see that if

$$
\sup _{r, t}\left\|\mathbf{s}^{E}(r, t)\right\| \leq \sqrt{2} \sigma_{Y}
$$

then shakedown occurs. It can be verified that the supremum in (34) is reached at $r=R$. Condition (34) can thus be rewritten as

$$
\left\|\mathbf{s}^{E}\left(R, t_{0}\right)\right\| \leq \sqrt{2} \sigma_{Y}
$$

where $t_{0} \in[0, T]$ is such that $\left\|\mathbf{s}^{E}\left(R, t_{0}\right)\right\|=\sup _{t} \| \mathbf{s}^{E}(R, t \|$. By Theorem 3 , condition (35) is a sufficient condition for shakedown. Theorem 4 shows that it is also necessary. Consider indeed the history defined by

$$
\mathbf{d}(\mathbf{x}, t)=\frac{1}{4 \pi R L} \frac{\mathbf{s}^{E}\left(R, t_{0}\right)}{\left\|\mathbf{s}^{E}\left(R, t_{0}\right)\right\|^{2}} \boldsymbol{\delta}(r-R)\left(\boldsymbol{\delta}\left(t-t_{0}\right)-\delta\left(t-t_{1}\right)\right)
$$

where $L$ is the length of the particle in the $\mathbf{e}_{z}$ direction and $\delta$ is the Dirac distribution. The time instant $t_{1}$ in $\sqrt{36}$ is defined by $t_{1}=\left(t_{0}+T / 2\right) \bmod T$. Observe in particular that $\mathbf{s}^{E}\left(R, t_{1}\right)=-\mathbf{s}^{E}\left(R, t_{0}\right)$. We have $\int_{0}^{T} \mathbf{d}(\mathbf{x}, t) d t=0$ hence $\int_{0}^{T} \mathbf{d}(\mathbf{x}, t) d t \in \mathscr{B}_{0}$. 
Moreover we have $\int_{\Omega} \int_{0}^{T} \sigma^{E}: \mathbf{d} d \Omega d t=1$. Using Theorem 4 with the history $\mathbf{d}$ in (36) shows that a necessary condition for shakedown is

$$
1 \leq \int_{\Omega} \int_{0}^{T} \mathscr{D}(\mathbf{d}(\mathbf{x}, t)) d \Omega d t
$$

For the Mises elasticity domain, the function $\mathscr{D}$ is given by $\mathscr{D}(\mathbf{d})=\sqrt{2} \sigma_{Y}\|\mathbf{d}\|$ provided that $\mathbf{d}$ is deviatoric. It follows that

$$
\int_{\Omega} \int_{0}^{T} \mathscr{D}(\mathbf{d}(\mathbf{x}, t)) d \Omega d t=\frac{\sqrt{2} \sigma_{Y}}{\left\|\mathbf{s}^{E}\left(R, t_{0}\right)\right\|} .
$$

Condition (37) is thus the same as (35). This shows that Condition $(35)$ is both a necessary and a sufficient for shakedown to occur.

Condition (35) can be rewritten as [18]

$$
H \leq H_{S D}(\tilde{\omega})
$$

where

$$
H_{S D}(\tilde{\omega})=2 \frac{\sqrt{6} \sigma_{Y}}{G|\tilde{a}|}\left(A(\tilde{\omega})+\sqrt{A^{2}(\tilde{\omega})-B(\tilde{\omega})}\right)^{-\frac{1}{2}}
$$

and

$$
A(\tilde{\omega})=3(1-v)^{2}+(\Re z-1-v)^{2}+(\mathfrak{I} z)^{2}, B(\tilde{\omega})=12(1-v)^{2}(\mathfrak{I} z)^{2} ;
$$

with

$$
\tilde{\omega}=\omega T_{0}, \quad z=\frac{\hat{R} J_{0}(\hat{R})}{J_{1}(\hat{R})} .
$$

Using the values of the constitutive parameters reported in Sect. 2.3, we obtain that

$$
H_{S D}(20)<100 \mathrm{~mol} / \mathrm{m}^{3}<H_{S D}(10)
$$

For a loading parameter $H=100 \mathrm{~mol} / \mathrm{m}^{3}, \sqrt{41}$ means that elastic shakedown occurs for $\omega=10 / T_{0}$ but not for $\omega=20 / T_{0}$. Those predictions agree with the finite element simulations shown in Fig. 1 and Fig. 2 In the case $\omega=10 / T_{0}$ corresponding to elastic shakedown, a result from Theorem 1 and Theorem 2 is that the $C-V$ response in the cyclic steady state coincide (up to a time-independent translation) with the cyclic $C-V$ response of the purely elasto-diffusive problem. The latter can be calculated in closed form and is given by

$$
C^{E}(t)=\frac{1}{2}(1+\sin \omega t), \quad \mu^{E}(t)=\frac{1}{4} \Re\left(e^{i \omega t} i z\right)
$$

The $C-V$ response provided by (42) is shown in Fig. 4(red line). The curve obtained form the finite element simulations of the diffusion-induced plasticity problem is shown as a blue curve in Fig. 4. It appears that the steady state in the $C-V$ plane is translated form the elasto-diffusive response as expected. 
Fig. 4 Charge-Voltage response for $H=100 \mathrm{~mol} / \mathrm{m}^{3}$, $\omega=10 / T_{0}$. Diffusion-induced plasticity (blue curve), pure elasto-diffusion (red curve).

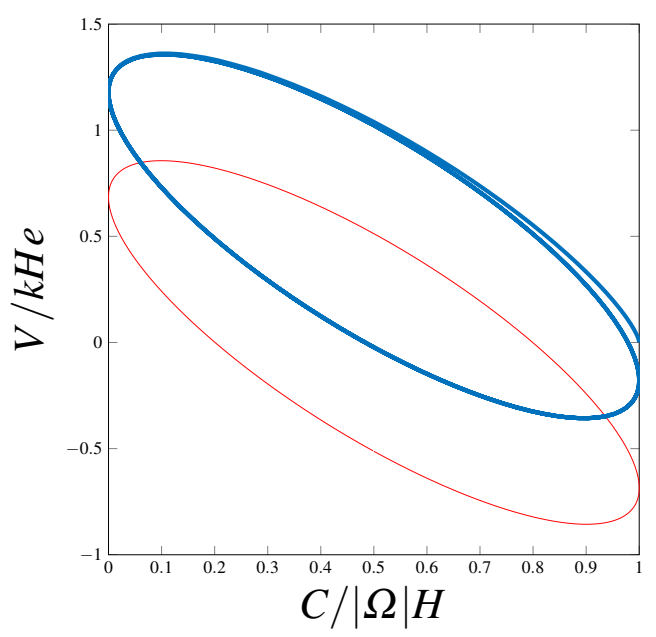

\section{References}

1. Brassart L., Zhao K. Suo Z. (2013) Cyclic plasticity and shakedown in high-capacity electrodes of lithium-ion batteries. Int. J. Solids. Struct. 49:1120-1129.

2. K. Dang Van, I. V. Papadopoulos (1999), Introduction to fatigue analysis in mechanical design by the multiscale approach. In: High-Cycle Metal Fatigue. Springer

3. O. Débordes, B. Nayroles, et al., Sur la théorie et le calcul à l'adaptation des structures élastoplastiques., J. Mecanique 15 ( 1976) 1-53.

4. Frederick, C., Armstrong, P., 1966. Convergent internal stresses and steady cyclic states of stress. J. Strain Anal. Eng. Des. 1, 154-159.

5. Halphen, B., 1978. Steady cycles and shakedown in elastic-viscoplastic and plastic structures. In: Structures et matériaux sous chargement cyclique. Association amicale des ingénieurs anciens éleves de l'E.N.P.C., pp. 203-229.

6. Johnson, C., Mercier, B., Nedelec, J. C., 1978. Convergence to a periodic solution in perfect plasticity. In: Structures et matériaux sous chargement cyclique. Association amicale des ingénieurs anciens éleves de l'E.N.P.C., pp. 253-255.

7. Klarbring,A., Barber, J.R., Spagnoli, A., Terzano, M., Shakedown of discrete systems involving plasticity and friction, Eur. J. Mech. A 64:160-164

8. Koiter WT (1960) General theorems for elastic-plastic solids. In: Progress in solid mechanics

9. Larché F., Cahn J. W. (1973) A linear theory of thermochemical equilibrium of solids under stress. Acta Metallurgica 21:1051-1063.

10. Maitournam H, Pommier B, Thomas JJ (2002) Détermination de la réponse asymptotique d'une structure anélastique sous chargement cyclique. C.R. Mecanique 330:703-708

11. Melan E (1936) Theorie statisch unbestimmter Systeme aus ideal-plastischen Baustoff. Sitz.Berl.Ak.Wiss. 145: 195-218

12. Nguyen QS (2003) On shakedown analysis in hardening plasticity. J. Mech. Phys. Solids $51: 101-125$

13. Opial, Z., 1967. Weak convergence of the sequence of successive approximations for nonexpansive mappings. Bull. Amer. Math. Soc. 73, 591-597.

14. Peigney M (2008) Recoverable strains in composite shape memory alloys, J. Mech. Phys. Solids 56:360- 375

15. Peigney $M$ (2010) Shakedown theorems and asymptotic behaviour of solids in non-smooth mechanics. Eur. J. Mech. A 29:784-793 
16. Peigney M (2014) On shakedown of shape memory alloys structures, Ann. Solid Struct. Mech. On shakedown of shape memory alloys structures 6:17-28

17. Peigney M (2014) Shakedown of elastic-perfectly plastic materials with temperaturedependent elastic moduli, J. Mech. Phys. Solids 71:112-131

18. Peigney M (2018) Cyclic steady states in diffusion-induced plasticity with applications to lithium-ion batteries, J. Mech. Phys. Solids 111:530-556

19. Peigney M (2020), Static and kinematic shakedown theorems in diffusion-induced plasticity, in preparation.

20. Peigney M, Stolz C (2001) Approche par contrôle optimal des structures élastoviscoplastiques sous chargement cyclique. C.R.Acad.Sci. Paris II 329:643-648

21. Peigney M, Stolz C (2003) An optimal control approach to the analysis of inelastic structures under cyclic loading. J. Mech. Phys. Solids 51:575-605

22. Pham, D. C., 2017. Consistent limited kinematic hardening plasticity theory and pathindependent shakedown theorems. Int. J. Mech. Sci. 130, 11-18.

23. Spiliopoulos KV, Panagiotou KD (2012) A direct method to predict cyclic steady states of elastoplastic structures. Comput. Methods Appl. Mech. Eng. 223:186-198

24. Symonds PS (1951) Shakedown in continuous media. J. Appl. Mech. 18:85-89 